\title{
Die Rolle des Internets im Radikalisierungsprozess einer jihadistischen Straftäterin - eine Einzelfallstudie
}

\author{
Dirk Baehr
}

Online publiziert: 8 . Oktober 2020

(C) Der/die Autor(en) 2020

Zusammenfassung Anhand der Einzelfallstudie des Radikalisierungsprozesses einer jihadistischen Straftäterin möchte dieser Beitrag die besondere Rolle des Internets bei Radikalisierungen von Extremisten aufzeigen. Die Straftäterin war in den Jahren 2007 bis 2010 einePropagandistin, die über das Internet mehrere terroristische Vereinigungen unterstützte, indem sie deren Videobotschaften online verbreitete. In der Einzelfallstudie wird aufgezeigt, wie sich die Jihadistin durch virtuelle Kontakte, Propagandaaktivitäten sowie ihre Zusammenarbeit mit zahlreichen Jihadisten im Ausland über das Internet radikalisierte.

Schlüsselwörter Radikalisierung · Internet · Jihadismus · Propaganda · Extremismus

\section{The Role of the Internet on Radicalization of a German Jihadist Offender: A Case Study}

\begin{abstract}
Based on the analysis of a case study this article raises the question about the role of the internet in accelerating the radicalization of a woman who joined the jihadist milieu in southern Germany in the mid 2000s. From 2007 to 2010 she was a jihadist activist on the internet who supported several terrorist groups by publishing videos and jihadi messages in blogs, video channels and web forums. The case study shows how the woman was radicalized through virtual contacts, interactions in virtual communities, as well as through her support of several jihadists abroad.
\end{abstract}

Bei diesem Beitrag handelt es sich um eine überarbeitete und konzentrierte Fassung einer früheren Studie des Autors (Baehr 2019), aus der einige Textstellen wortwörtlich übernommen wurden.

D. Baehr $(\bowtie)$

Universität zu Köln, Köln, Deutschland

E-Mail: dirk.baehr@posteo.de 
Keywords Radicalisation · Jihadism $\cdot$ Terrorism $\cdot$ Internet $\cdot$ Social Media $\cdot$ Al Qaeda

\section{Einleitung}

Die vorliegende Studie befasst sich mit der Rolle des Internets bei Radikalisierungsprozessen von Jihadist*innen in Deutschland. In der Studie greift der Autor die Ergebnisse seiner Forschungsarbeit über die Ursachen von Radikalisierungen jihadistischer Straftäter*innen auf. Für die Forschungsarbeit wurden die Radikalisierungsprozesse von neun Jihadisten*innen analysiert, die entweder in Deutschland geboren sind oder seit ihrer Kindheit in Deutschland leben. Bei den jungen Straftäter*innen handelt es sich entweder um jihadistische Extremist*innen, die Werbung für terroristische Bewegungen betrieben sowie diese durch das Sammeln von Spendengeldern unterstützt haben oder es handelt sich um Mitglieder einer terroristischen Vereinigung. Das empirische Datenmaterial zu den Lebensläufen der neun Jihadist*innen ist bei der Beobachtung von sieben Gerichtsverfahren gewonnen worden, in denen die betreffenden Jihadist*innen angeklagt und verurteilt wurden (Baehr 2019).

Der Autor konnte durch die Auswertung des empirischen Datenmaterials die unterschiedlichen Stufen der Radikalisierung der neun Jihadist*innen in der Forschungsarbeit aufzeigen. Zuerst konzentrierte er sich in der Arbeit auf das Leben vor der Radikalisierung der jihadistischen Straftäter*innen. Anhand des empirischen Datenmaterials aus den Gerichtsverhandlungen war es möglich, bestimmte Belastungsmomente oder vorgelagerte Ereignisse zu identifizieren, mit denen aufgezeigt werden konnte, dass in der Kindheit oder Jugend der Täter*innen signifikante Probleme aufgetreten waren, die sich negativ auf ihre weitere Sozialisation auswirkten. Mit einem biographisch orientierten Forschungsansatz erklärt der Autor die Belastungsmomente in der Kindheit oder Jugend und zeigt dabei auf, weswegen diese den Einstieg in die Radikalisierung befördert haben könnten. Des Weiteren geht der Autor der Forschungsfrage nach, wie Radikalisierungsprozesse ausgelöst werden und wie der Einstieg in das extremistische Milieu erfolgt. Konkret stellt er sich die Fragen: Wann, wo und unter welchen Umständen begann die Radikalisierung der neun Jihadist*innen? Wieso fanden sie jihadistische Bewegungen wie Al-Qaida attraktiv? Wie sind sie ins salafistische bzw. jihadistische Milieu hineingeraten und wie verliefen die ersten Stufen der Radikalisierung? Anhand des in den Gerichtsverfahren gewonnenen Datenmaterials ließen sich die Radikalisierungsprozesse von zwei Jihadisten rekonstruieren. Bei den anderen jihadistischen Straftäter*innen gab es nur vereinzelte Hinweise, die erklärten, welche individuellen, psychologischen, situationsbedingten, gruppendynamischen, ideologischen oder sozialen Faktoren maßgeblich zu ihrer Radikalisierung beigetragen hatten (Baehr 2019).

Der Autor untersuchte in seiner Forschungsarbeit auch den Einfluss des Internets und der Sozialen Medien auf Radikalisierungsprozesse der neun jihadistischen Straftäter*innen. Durch die Auswertung des empirischen Datenmaterials in den Gerichtsverfahren ließ sich nachweisen, dass bei fünf der neun verurteilten Jihadist*innen das Internet eine besonders wichtige Rolle in den Radikalisierungsprozessen spielte. 
Alle fünf Jihadist*innen wurden durch jihadistische Inhalte im Internet stark beeinflusst. Sie nutzten fast täglich jihadistische Internetseiten, Blogs und Webforen. Dabei konsumierten sie auch extremistische Videos, mit ideologischen Botschaften oder Berichten über Kampfhandlungen, bei denen Verletzte und Tote zu sehen waren. Durch die intensive Beschäftigung mit der jihadistischen Propaganda übernahmen sie immer mehr den Deutungsrahmen des bewaffneten Jihad als so genannte Verteidigungsmaßnahme gegen den Westen. Vier der neun verurteilten Jihadist*innen gründeten eigene Videokanäle, auf denen sie Videos von jihadistischen Bewegungen veröffentlichten, um die Idee des bewaffneten Kampfes gegen die Ungläubigen zu unterstützen. Daneben verbreiteten sie auch selbstverfasste Beiträge, in denen jihadistisches Gedankengut vermittelt und für eine aktive Teilnahme am bewaffneten Kampf gegen Deutschland bzw. Bundeswehrsoldat*innen in Afghanistan geworben wurde (Baehr 2019).

In der hier vorliegenden Studie werden die Ergebnisse des Radikalisierungsprozesses der einzigen Frau, die in der Forschungsarbeit analysiert wurde, vorgestellt, da in ihrem Fall empirisches Datenmaterial vorhanden war und ausgewertet werden konnte, welches die Rolle des Internets sehr anschaulich aufdeckt. Dem Autor geht es nicht darum, eine genderbezogene Perspektive in der Einzelfallstudie zu leisten, eher möchte er im Kontext der Radikalisierungs- und Terrorismusforschung den Einfluss des Internets bei Radikalisierungen von jihadistischen Extremist*innen aufzeigen.

Aus datenrechtlichen Gründen wurde der Name der jihadistischen Straftäterin anonymisiert und geändert. Im Folgenden wird die Frau Fatma genannt. Fatma gehörte unter den neun analysierten Fällen zu einer der aktivsten Propagandist*innen, da sie über das Internet mehrere terroristische Vereinigungen (Al-Qaida, Islamische Jihad Union (IJU) und Deutsche Taliban Mudschahideen) unterstützte. Sie verbreitete deren extremistische Videobotschaften online, produzierte zugleich eigene Videos und Textbeiträge - u. a. auch für die Deutschen Taliban Mudschahideen (DTM) und erlangte dadurch in der virtuellen Welt einen gewissen Kultstatus. Anhand der Einzelfallstudie soll aufgezeigt werden, wie sich Fatma durch virtuelle Kontakte, Gespräche in Chatrooms, ihre Propagandaaktivitäten sowie durch die Zusammenarbeit mit Jihadist*innen im Ausland über das Internet radikalisierte.

\section{Forschungsfrage und Methodik}

Der Autor möchte mit der hier vorliegenden Studie die beiden Fragen beantworten: Wie verlaufen die Radikalisierungsprozesse von deutschen Jihadist*innen im Internet und den Sozialen Medien und welche Rolle spielen dabei das Internet und die Sozialen Medien? Das Ziel der Studie ist es, anhand der Einzelfallstudie aufzuzeigen, wie sich Kontakte, Kommunikation und Interaktionsprozesse in virtuellen Gemeinschaften radikalisierungsfördernd auswirken. Es soll überprüft werden, welche Einflüsse und Effekte sowohl die virtuellen Kontakte zu einzelnen Extremist*innen bzw. terroristischen Vereinigungen sowie die Interaktionen in den virtuellen Gemeinschaften als auch die Propagandabotschaften von jihadistischen Internetaktivist*innen bzw. Gruppen auf Radikalisierungsprozesse haben. 
Um die Forschungsfrage zu beantworten, analysiert der Autor in einer Einzelfallstudie den Radikalisierungsprozess einer jihadistischen Straftäterin. In der qualitativen Terrorismusforschung sind Fallstudien die am häufigsten angewendete Methode zur Ursachenanalyse von Terrorismus und Radikalisierungen. Durch die intensive Analyse einzelner Fälle erlangen Radikalisierungsforscher*innen die Fähigkeit, Veränderungen im Sozialisationsprozess der sich radikalisierenden Personen zu entdecken bzw. zu erfassen (Hamm und Spaaij 2017) und einschneidende Ereignisse im Leben der Extremist*innen zu rekonstruieren (Odă̆ et al. 2020), um bestimmte Faktoren für die Ursache der Radikalisierungen zu identifizieren. Dementsprechend eignen sich Fallstudien zur Ursachenanalyse von Radikalisierungen von jihadistischen Extremist*innen besonders gut, weil sie helfen, komplexe Interaktionsprozesse der sich radikalisierenden Personen (mit anderen Extremist*innen) und ihre Dynamiken zu verstehen, besondere individuelle Charakteristika und grundsätzlich gemeinsame Merkmale von extremistischen Straftäter*innen aufzudecken und bestimmte Faktoren, die die Radikalisierungen verursachen, zu erkennen (O'Connor et al. 2017; Böckler et al. 2017). Folgende Aspekte sind in der Einzelfallstudie von besonderem Interesse:

1. Gibt es besondere Ereignisse in der Biografie der jihadistischen Straftäterin, die als zentraler Auslöser der Radikalisierung oder als Wendepunkt in ihrem Leben angesehen werden können und inwieweit spielt dabei das Internet eine Rolle?

2. Was hat die Straftäterin im Internet gemacht? Wirkte sich die extremistische Propaganda radikalisierungsfördernd auf die Straftäterin aus? Welche Form bzw. welches Mittel der Propaganda wirkte sich am intensivsten auf die Einstellungen der Straftäterin aus? Übernahm sie durch den Konsum der extremistischen Internetpropaganda die Deutungsrahmen und Narrative der jihadistischen Bewegungen?

3. Entwickelte sich durch ihre virtuellen Kontakte und Verbindungen eine größere virtuelle Gemeinschaft, die die Radikalisierung der Straftäterin verstärkte? Oder gab es virtuelle Kontakte und Interaktionen mit bestimmten Personen, die maßgeblich die Radikalisierung beeinflussten? Wie entwickelte sich ein Zugehörigkeitsgefühl in der virtuellen Gemeinschaft und befördern solche Interaktionen die Radikalisierung der Straftäterin?

Anhand von einzelnen Fallanalysen ist es allerdings schwierig, grundsätzliche Erklärungsansätze für die Ursachen und Faktoren von Radikalisierungsprozessen zu geben, da bei jedem Einzelfall, bei jeder untersuchten Person, verschiedene individuelle Entwicklungswege identifiziert werden und diverse Faktoren die Radikalisierungen ausgelöst bzw. verursacht haben können. Dadurch ist es nicht möglich, allgemeine theoretische Erklärungsansätze zu entwickeln, weil sich die Forschungsergebnisse einer Einzelfallstudie kaum generalisieren lassen. Obwohl die Ursachen der Radikalisierungen von Person zu Person variieren, ist es möglich, wiederkehrende Muster in den Prozessen zu erkennen, die zeigen, dass einerseits bestimmte Radikalisierungsfaktoren immer wieder in Fallanalysen auftreten und andererseits diese Faktoren zumeist nur in bestimmten Stufen oder Phasen der Radikalisierung auftreten. So sind zahlreiche Modelle von Radikalisierungs- bzw. Terrorismusforscher*innen entwickelt worden (Moghaddam 2005; Silber und Bhatt 2007; McCauley und Moskalenko 2017), die erklären, dass sich Radikalisierungen von Terro- 
rist*innen stufen- oder phasenweise vollziehen, die zu emotionalen und kognitiven Einstellungs- und Verhaltensveränderungen führen und am Ende zur terroristischen Gewalt beitragen. Daneben verdeutlichen die Modelle allerdings auch, dass in den jeweiligen Stufen oder Phasen der Radikalisierung oft nur bestimmte Faktoren eine zentrale Rolle spielen.

Ähnlich ist es auch bei der Rolle des Internets und der Sozialen Medien bei Radikalisierungen von Jihadist*innen. Auch hier treten wiederkehrende Muster in den Radikalisierungsprozessen auf, die den Autor veranlasst haben, die Ergebnisse der Einzelfallanalyse mit den internationalen Forschungsergebnissen über die Rolle des Internets in Radikalisierungen zu vergleichen. Diese Ergebnisse zeigten, dass in verschiedenen Stufen oder Phasen bestimmte Faktoren und Mechanismen bzw. Dynamiken auftraten, die im Radikalisierungsprozess von Fatma ebenfalls identifiziert wurden.

Dies veranlasste den Autor, ein fünfstufiges Modell der Online-Radikalisierung zu entwickeln. Das Modell bietet eine Grundlage für das Verständnis von Radikalisierungsprozessen, die besonders stark durch das Internet und die Sozialen Medien beeinflusst wurden. Um die besonderen Eigenschaften des Internets und der Sozialen Medien bei Radikalisierungen verstehen zu können, ist ein Modell der OnlineRadikalisierung hilfreich, da dieses verständlich aufzeigt, welche besondere Rolle virtuellen Kontakten, Kommunikation und Interaktionen zwischen der sich radikalisierenden Person und den anderen (Internet-)Akteuren und den daraus entstehenden Dynamiken zukommt. Dabei kann sehr anschaulich dargelegt werden, inwiefern diese Dynamiken zu den sich verstärkten oder auch beschleunigten Radikalisierungen mit beitragen. Zugleich wird durch das Modell deutlich, dass es verschiedene Stufen oder Phasen gibt, in denen bestimmte Dynamiken und Faktoren die Radikalisierungen beeinflussen, diese die Prozesse verstärken oder beschleunigen und somit maßgeblich weiter intensivieren. Entscheidend ist, dass das Modell aufzeigen soll, dass sich Radikalisierungen im Internet auch stufen- oder phasenweise vollziehen und diese durch das Internet verursachte Prozesse viele miteinander verbundene Faktoren beinhalten, die zu verschiedenen Stufen oder Phasen auftreten und bestimmte Wirkungen auf die radikalisierende Person haben. Ob und inwiefern das aus der Einzelfallanalyse geschlossene Modell eine allgemeinere Gültigkeit beanspruchen kann, muss sich aus künftigen Analysen von Fallstudien erweisen. Das Modell ist aber als Werkzeug für zukünftige Analysen von Einzelfallstudien besonders hilfreich, um den Einfluss durch das Internet und die Sozialen Medien auf Radikalisierungsprozesse einordnen und erklären zu können. 


\section{Aktueller Forschungsstand über die Rolle des Internets und der Sozialen Medien in Radikalisierungsprozessen von Jihadisten}

Seit Ende der 1990er-Jahre nutzen jihadistische Bewegungen das Internet, um den Kampf um die Herzen und den Verstand ${ }^{1}$ potenzieller Anhänger zu führen (Stout 2008). Der heutige Chef der Al-Qaida, Ayman al-Zawahiri, behauptete in einem 2005 öffentlich bekannt gewordenen Brief an Abu Musab al Zarqawi, dass der Krieg gegen die Ungläubigen zur Hälfte in den Medien stattfinde. Auch der jemenitischamerikanische Ideologe Anwar al-Awlaki betonte wenig später in einem Vortrag mit dem Titel „Die Schlacht um die Herzen und den Verstand“ (al-Awlaki 2008), dass der Krieg gegen den Westen nicht nur eine militärische Schlacht sei, sondern auch ein ideologischer Kampf zwischen dem Westen und der muslimischen Welt, der im Internet geführt werden müsse. Aus diesem Grund verstärkten jihadistische Bewegungen wie Al-Qaida seit 2006/2007 die Propagandaarbeit im Internet, um ihre jihadistische Ideologie global verbreiten und neue Anhänger für ihren Kampf mobilisieren zu können (Whitlock 2008).

Ab Mitte der 2000er Jahre wandten sich Wissenschaftler*innen vermehrt der Frage nach der Wirksamkeit der Internetpropaganda von Al-Qaida zu. Zu den Ersten, die sich mit der Thematik beschäftigten, gehörten die norwegische Politikwissenschaftlerin Hanna Rogan (Rogan 2006, 2007) und der israelische Medienwissenschaftler Gabriel Weimann (Weimann 2006a, b, c, d). Rogan argumentiert, dass das Internet für jihadistische Bewegungen an großer Bedeutung gewonnen habe, weil es ihnen neue Möglichkeiten zur Verbreitung ihrer Inhalte und zur Intensivierung ihrer Propagandaarbeit biete. Ohne das Internet könnten diese Bewegungen nicht jene enorme Aufmerksamkeit und Publizität erlangen, die ihnen ihre selbst produzierten Videos aus den Kriegsgebieten verschaffen (Rogan 2006). Dementsprechend hilft ihnen das Internet erheblich dabei, ein großes und „,bedeutendes Publikum zu erreichen“ (Rogan 2006, S. 32; eigene Übersetzung). Auch Weimann sieht im Internet eines der entscheidenden Werkzeuge des postmodernen Terrorismus. Durch die mit dem Internet entstandenen neuen Kommunikationstechnologien, so Weimann, können Terrorist*innen Anschläge planen, vorbereiten und ausführen, ohne dabei geographisch an ein bestimmtes Territorium gebunden oder finanziell von einem bestimmten Staat abhängig zu sein. Das Internet ermöglicht es den Terrorist*innen von Al-Qaida, dem Islamischen Staat oder anderen jihadistischen Gruppierungen also, ihre Propaganda weltweit zu verbreiten. Dabei sind sie in der Lage, ihre Ideologie oder ihre jihadistischen Deutungsrahmen einem globalen Publikum zu vermitteln, mit dem sie ansonsten kaum oder gar nicht in Kontakt treten könnten. Durch das Internet können Jihadist*innen neue Anhänger*innen rekrutieren sowie mit Sympathisant*innen, die über die ganze Welt verstreut leben, kommunizieren (Weimann 2006a, S. 20).

\footnotetext{
1 Das Konzept des „Winning the Hearts and Minds“ wurde ursprünglich vom britischen General Gerald Templer vor dem Hintergrund der malaysischen Unabhängigkeitskämpfe entwickelt (Siehe hierzu Hack 2009). Es handelt sich um eine militärische Strategie, die in Kombination mit wirtschaftlichen, sozialen und politischen Maßnahmen umgesetzt wurde. Die britische Militärstrategie haben jihadistische Strategen, die Al-Qaida nahestanden, übernommen.
} 
Seit Mitte der 2000er Jahre beschäftigt sich auch Maura Conway mit der Propaganda von terroristischen Bewegungen im Internet. In einem Aufsatz von 2006 stellt die irische Terrorismusexpertin zunächst fest, dass bisher zwar nicht nachgewiesen werden konnte, dass die Anzahl der Terroranschläge infolge der Internetnutzung durch jihadistische Gruppierungen gestiegen wäre. Zugleich aber macht sie deutlich, dass Terrorist*innen durch das Internet befähigt seien, Spendengelder zu sammeln, mit ihrer Propaganda ein Massenpublikum zu erreichen und dadurch neue Anhänger zu mobilisieren bzw. zu rekrutieren (Conway 2006, S. 25). Für Conway wie auch zahlreiche andere Expert*innen liegt das Besondere am Internet darin, dass dort virtuelle Milieus (Conway 2010, 2016, S. 6) bzw. virtuelle Gemeinschaften (Radlauer 2007; Janbek und Prado 2012; Bowman-Grieve 2013) entstehen können, die - wenn dort extremistische Ansichten artikuliert oder verbreitet würden - bei ihren Teilnehmer*innen potenziell den Effekt haben, Radikalisierung zu fördern. Radikalisierungsforscher*innen, die ihren Forschungsschwerpunkt auf das Internet bzw. auf extremistische Aktivist*innen im Internet richten, entwickelten daher den Begriff der Online-Radikalisierung und verstehen darunter:

Online radicalisation is here conceived as a process whereby individuals, through their online interactions and exposure to various types of Internet content, come to view violence as a legitimate method of solving social and political conflicts. (Bermingham et al. 2009, S. 2)

Auch Conway sieht in den zahlreichen Fallbeispielen, in denen jihadistische Terrorist*innen durch extremistische und gewaltverherrlichende Inhalte im Internet beeinflusst wurden, einen Beleg dafür, dass das Internet eine zentrale Rolle bei Radikalisierungsprozessen spielt. Zu den wichtigsten Fällen zählt sie die Radikalisierung von Arid Uka, Hussain Osman und Nidal Hasan. Am 2. März 2011 erschoss der damals 21-jährige Uka zwei amerikanische Soldaten und verletzte zwei weitere am Frankfurter Flughafen. Osman gehörte zu den Attentätern der Terroranschläge vom 7. Juli 2005 in London, während Hasan als jihadistischer Einzeltäter 2009 in dem amerikanischen Militärcamp Fort Hood 13 Soldaten erschoss und über 30 weitere verwundete (Conway 2010, S. 12).

Angesichts der weithin anerkannten Bedeutung der Internetkommunikation für den gegenwärtigen jihadistischen Terrorismus hat Conway den Mangel an sozialwissenschaftlichen Studien kritisiert, die den Einfluss des Internets auf Prozesse der Radikalisierung in den Jihadismus aufzeigen und beweisen würden (Conway 2010, S. 17). Dies hängt unter anderem damit zusammen, dass es für Wissenschaftler*innen schwierig sei, die Rolle des Internets bei Radikalisierungsprozessen zu belegen, da es nur wenige gründlich erforschte Fallbeispiele gebe, die aufzeigen, wie das Internet die Radikalisierung von Jihadisten (mit)befördert habe. Laut Conway liegen hier die maßgeblichen Versäumnisse der Forschung. Es gebe zwar ausreichend Anhaltspunkte, die die Bedeutung des Internets in Radikalisierungsprozessen aufzeigen würden, dabei seien jedoch nur wenige sozialwissenschaftliche Untersuchungen erschienen, die auf empirische Daten zurückgreifen (Conway 2010, S. 18). Eine ähnliche Kritik äußern auch Paul Gill et al. in ihrer 2015 erschienen Studie What are the roles of the Internet in Terrorism? für das VOX-Pol-Netzwerk (Gill et al. 2015, S. 5). Sie kritisieren den Mangel an datenbasierten Forschungsarbeiten, 
die Aufschluss über den Einfluss von extremistischen Internetseiten geben. So verweisen sie darauf, dass sich nur 6,5\% der 200 wissenschaftlichen Untersuchungen, die sie in ihrer Studie einbezogen haben, auf empirisches Datenmaterial beziehen; und sogar nur $2 \%$ auf primäre Daten - also Interviews mit Terrorist*innen oder Gerichtsunterlagen zu terroristischen Fällen. Die meisten Daten resultieren aus der Auswertung von extremistischen Internetseiten und gelten daher als sekundäre Daten. In diesem Mangel an primären Daten sehen die Forscher*innen ein wesentliches Hindernis bei der Aufgabe, die komplexen Ursachen von Radikalisierungsprozessen und die Bedeutung des Internets in diesem Zusammenhang adäquat zu erklären (Gill et al. 2015, S. 5-7).

Die Problematik der Ursachenanalyse ist jedoch tiefgreifender als es Conway sowie Gill et al. in ihren Arbeiten schildern. Denn es gibt nicht nur einen Mangel an primären Daten für die Erforschung von Radikalisierungsprozessen, sondern es ist für die Terrorismusforscher*innen generell schwierig, primäre Daten zu erhalten, die aufzeigen, wie Terrorist*innen das Internet nutzen und wie sie mit anderen in der virtuellen Welt kommunizieren. Der Großteil der primären Daten, die aus Gerichtsunterlagen gewonnen werden können, lassen zumeist nur Aussagen über jihadistische Internetseiten und die darauf veröffentlichten Inhalte zu, die die jihadistischen Terroristen entweder selber dort publiziert oder die sie rezipiert haben. Dabei wäre es bedeutend wichtiger, die Kommunikation zwischen Terrorist*innen im Internet verfolgen zu können, um auf diese Weise die radikalisierende Wirkung der virtuellen Kommunikation und Interaktionen nachvollziehen und untersuchen zu können.

Aktuell gibt es nur wenige Studien, in denen empirisches Datenmaterial für die Analyse der Rolle des Internets in Radikalisierungsprozessen verwendet wurde. Dies sind zum einen zwei umfangreiche Studien von Lorenzo Vidino mit verschiedenen Ko-Autoren (Vidino et al. 2015; Vidino und Hughes 2015) die RAND-Studie von Ines Behr et al. (von Behr et al. 2013), ein Arbeitspapier von Benjamin Ducol et al. (Ducol et al. 2016) und die bereits genannte Untersuchung von Gill et al. sowie Paul Gills Dissertation (Gill et al. 2015; Gill 2015). Daneben gibt es noch wissenschaftliche Aufsätze, die sich u.a. auch auf empirisches Datenmaterial stützen. Hierzu zählen die Beiträge von Gill et al. (Gill et al. 2013, 2017) sowie fünf Einzelfallanalysen von verschiedenen Autor*innen (Anzalone 2010, 2012; Boyle 2013; Mastors und Siers 2014; Pearson 2015). Staatliche (Sicherheits-)Behörden veröffentlichen gelegentlich auch Fallstudien, die sich zu den Internetaktivitäten von Jihadist*innen äußern (siehe beispielsweise Senate Committee on Homeland Security and Governmental Affairs 2012).

Bevor die Ergebnisse der Studie zum Fall Fatma dargelegt werden, sollen an dieser Stelle ein paar wichtige Forschungsergebnisse zu Online-Radikalisierungen vorgestellt werden. Das Forscherteam um Vidino analysierte in seiner Studie Terrorist Chatter (Vidino et al. 2015) die Profile von sieben jihadistischen Internetaktivisten, um virtuelle Kommunikations- oder Interaktionsmechanismen zu erforschen und herauszufinden, ob sich diese Mechanismen radikalisierungsfördernd auswirken. Die sieben untersuchten Jihadisten, die alle in den USA geboren oder aufgewachsen waren, betrieben zwischen Mitte der 2000er Jahre und Anfang der 2010er Jahre jihadistische Propaganda im Internet. Drei von ihnen wurden wegen der Werbung 
für terroristische Vereinigungen strafrechtlich verurteilt. Zwei weitere schlossen sich im Ausland einer jihadistischen Bewegung an und betrieben von dort weiter Propaganda im Internet. Beide kamen bei Kämpfen bzw. bei einem Drohnenangriff ums Leben (Vidino et al. 2015, S. 2-6). Vidino und sein Team stellten bei den sieben jihadistischen Internetaktivisten die verschiedensten Ansichten und Interessen fest. Die unterschiedlichen Ansichten führten sie darauf zurück, dass einige schon längere Zeit im Internet aktiv waren und dort jihadistische Inhalte verbreiteten, während andere sich erst im Frühstadium ihres Radikalisierungsprozesses befanden. Auch die Interessen variierten von Person zu Person: Nur drei der untersuchten Jihadisten interessierten sich vor allem für religiöse Themen. Andere suchten hingegen Internetbeiträge über politische und soziale Themen. Was sie alle in ähnlicher Weise antrieb, war die intensive Suche nach Informationen. Dabei scheint es ihnen wichtig gewesen zu sein, dass die Texte oder Videos im Internet ihre eigenen Positionen bestätigten. Die Studie belegt, dass die Kommunikation und Interaktion im Internet ein wesentlicher Faktor in der Einstiegsphase von Radikalisierungen sein kann, weil die Internetaktivisten ,radikale Einstellungen haben und in einigen Fällen diejenigen herausfordern, deren Meinungen sich von ihren unterschieden“ (Vidino et al. 2015, S. 74, eigene Übersetzung). Das Internet fungiert demnach als ein Echoraum, in dem sich die radikalen Ansichten einer Person immer weiter verstärken können.

Die zweite Studie, die sich mit den Ursachen von Online-Radikalisierungen beschäftigte, ist die Untersuchung von Ines von Behr et al. mit dem Titel Radicalisation in the Digital Era. Die Forscher*innen analysierten anhand empirischer Daten die Ursachen der Radikalisierung von 15 Jihadist*innen und die Bedeutung des Internets für ihre Radikalisierung. 9 der 15 Jihadist*innen wurden von britischen Gerichten wegen terroristischer Aktivitäten strafrechtlich verurteilt. In der Studie stellten die Forscher*innen anhand einer Analyse der vorhandenen Forschungsliteratur fünf Hypothesen zu der Frage auf, weswegen das Internet erheblichen Einfluss auf die Radikalisierung jihadistischer Terrorist*innen hat (von Behr et al. 2013, S. 12-13). Ihre Hypothesen lauten wie folgt:

1) Das Internet erzeugt bedeutend mehr Möglichkeiten, um sich oder andere Personen zu radikalisieren; 2) das Internet fungiert wie ein Resonanzraum (,echo chamber"); 3) das Internet beschleunigt Radikalisierungsprozesse; 4) das Internet ermöglicht die Radikalisierung von Menschen, ohne dass diese in physischen Kontakt mit Angehörigen der jeweiligen terroristischen Bewegung kommen müssen; 5) das Internet schafft neue Gelegenheiten für sogenannte Selbstradikalisierungen (von Behr et al. 2013, S. 16-21).

Die Hypothesen wurden von den Forscher*innen mit den Ergebnissen und Rückschlüssen aus dem empirischen Datenmaterial verglichen und auf ihre Plausibilität und Richtigkeit überprüft. Dabei stellten sie fest, dass die primären Daten die erste Hypothese bestätigten. Sie sahen es als erwiesen an, dass das Internet mehr Gelegenheiten schafft, dass sich jemand radikalisiert. Alle 15 Fälle, die in der Studie ausgewertet wurden, zeigten, dass das Internet die entscheidende Ressource war, um Informationen über jihadistische Bewegungen sowie jihadistisches Propagandamaterial zu erhalten und mit anderen Jihadist*innen in Kontakt zu kommen sowie zu kommunizieren (von Behr et al. 2013, S. 24-27). Die zweite Hypothese wurde ebenfalls bestätigt, da die Jihadist*innen in den meisten Fällen Diskussionen in den 
Webforen führten und Gleichgesinnte im Internet trafen, wodurch sie die Möglichkeit hatten, ihre extremistischen Positionen zu bekräftigen (von Behr et al. 2013, S. 27-28). Die dritte Hypothese ließ sich hingegen nicht verifizieren, da es keine genauen Angaben darüber gab, wie viele Stunden die Jihadist*innen am Tag im Internet surften, ob sie nur im Internet Kontakte zu anderen Jihadist*innen hatten oder ob sie sich auch durch Face-to-Face-Kontakte radikalisierten. Die Forscher*innen kamen deshalb zu dem Ergebnis, dass das Internet in den meisten Fällen die Radikalisierungen zwar erleichtert, aber nicht notwendigerweise beschleunigt habe (von Behr et al. 2013, S. 28). Dass das Internet Radikalisierungen ohne physische Kontakte ermögliche, ließ sich in der Studie ebenfalls nicht bestätigen. In den meisten Fällen waren die Jihadist*innen sowohl im Internet als auch offline aktiv, woraus die Forscher*innen schlossen, dass die Kontakte und Interaktionen in der realen Welt ebenfalls eine wesentliche Rolle bei den Radikalisierungen gespielt haben dürften. Auch die letzte Hypothese wurde von dem Forscherteam als nicht erwiesen erachtet, weil die von ihnen ausgewerteten empirischen Daten dafür sprachen, dass sich die Mehrheit der 15 Jihadist*innen sowohl durch virtuelle Kommunikation als auch durch Kontakte und Interaktionen in der realen Welt radikalisiert hatte (von Behr et al. 2013, S. 16-20). In aller Kürze zusammengefasst kamen von Behr und ihre Kolleg*innen zu dem Ergebnis, dass das Internet einer von vielen Faktoren ist, der Radikalisierungen verursacht oder befördert, ohne sie notwendigerweise zu beschleunigen (von Behr et al. 2013, S. 33).

Neben internationalen Forschungsbeiträgen sind in den letzten Jahren auch in Deutschland Studien erschienen, die empirische Daten genutzt haben, um die Rolle des Internets in den Radikalisierungsverläufen von deutschen Jihadist*innen zu untersuchen. Aktuell gibt es acht wissenschaftliche Forschungsarbeiten, die sich in ihren Analysen auf empirisches Datenmaterial stützen. Einer dieser Aufsätze stammt von dem Psychologen und Gerichtsgutachter Norbert Leygraf, der auf die (psychologischen) Faktoren der Radikalisierung bei sieben deutschen bzw. in Deutschland lebenden jihadistischen Straftäter eingeht, ohne sich jedoch explizit mit der Rolle des Internets zu beschäftigen (Leygraf 2014). In vier weiteren Studien befassen sich mehrere Forscherteams mit dem Fall des Frankfurter Einzeltäters Arid Uka und verweisen dabei auch auf die besondere Rolle des Internets in diesem Radikalisierungsprozess (Böckler et al. 2015; Malthaner und Lindekilde 2017) oder mit den Radikalisierungsprozessen von Anis Amri und den sogenannten Kofferbombern (Böckler et al. 2017; O'Connor et al. 2018). Die anderen Beiträge versuchen entweder anhand quantitativer Forschungsmethoden die radikalisierende Wirkung von Propaganda im Internet zu erfassen (Rieger et al. 2013; Schmitt et al. 2017) oder mit der Analyse umfangreichen Datenmaterials aus Chats von einem deutschen Dschihadisten auf WhatsApp die radikalisierende Wirkung virtueller Kommunikation aufzuzeigen (Kiefer et al. 2018). Neben den empirischen Studien sind noch weitere Aufsätze erschienen, die entweder einen Überblick über das Forschungsfeld geben (Dienstbühl und Weber 2014; Neumann et al. 2018; Inan 2012; Neumann 2018; Knipping-Sorokin und Stumpf 2018; Odă̆ et al. 2020) oder besondere Aspekte der Online-Radikalisierung hervorheben (Baugut und Neumann 2019; Baaken und Schlegel 2017; Böckler und Allwinn 2017; Christoph 2015; Frischlich et al. 2015; Jukschat und Kudlacek 2017; Rieger et al. 2019). 
Den ausführlichsten Bericht über die Ursachen der Radikalisierung deutscher Jihadist*innen hat das Bundeskriminalamt (BKA) in Kooperation mit dem Bundesamt für Verfassungsschutz (BfV) und dem Hessischen Informations- und Kompetenzzentrum gegen Extremismus (HKE) verfasst (Ständige Konferenz der Innenminister und -senatoren der Länder 2016). In der behördlichen Untersuchung, deren aktuellste Fassung aus dem Frühjahr 2016 stammt, wurden die primären Daten von 677 Jihadist*innen ausgewertet, die seit 2013 nach Syrien ausgereist sind und sich dort vermutlich einer ausländischen terroristischen Vereinigung angeschlossen haben. Die Analyse der Sicherheitsbehörden hat sich dabei auch mit der Rolle des Internets in Radikalisierungsprozessen befasst. Anhand der empirischen Daten fanden sie heraus, dass bei 201 Personen das Internet zu Beginn der Radikalisierung besonders relevant war. Bei weiteren $20 \%$ scheint das Internet hingegen erst im weiteren Verlauf der Radikalisierung entscheidend gewesen zu sein. Zudem ist den Behörden bekannt, dass $47 \%$ der Jihadist*innen in sozialen Netzwerken aktiv waren oder noch sind. In sehr wenigen Fällen $(8 \%)$ haben sich Jihadist*innen ausschließlich durch das Internet radikalisiert, also ohne mit anderen Aktivist*innen in physischen Kontakt getreten zu sein. Trotz der stichhaltigen empirischen Daten kamen die Analyst*innen der Sicherheitsbehörden in den vergangenen Jahren meistens zu dem Ergebnis, dass das Internet eine untergeordnete Rolle in Radikalisierungsprozessen von deutschen Jihadist*innen spielte. Erst in der neuesten Berichtsversion vertreten sie die Ansicht, dass der Radikalisierungsfaktor des sozialen Umfeldes an Bedeutung verloren und das Internet immer mehr an Einfluss auf solche Prozesse gewonnen habe.

Mit Blick auf radikalisierungsrelevante Einflussfaktoren zeichnen sich für die Gruppe der im letzten Jahr Ausgereisten [...] auffällige Veränderungen ab: Ein Bedeutungszuwachs des Internets $(55 \%$ gegenüber $38 \%$ bei den vor Ausruf des Kalifats Ausgereisten) korrespondiert mit einer geringeren Bedeutung des sozialen Nahraums innerhalb des Radikalisierungsprozesses (59\% gegenüber $71 \%$ ). (Ständige Konferenz der Innenminister und -senatoren der Länder 2016, S. 19-20, S. 49)

Der Einfluss des Internets war allerdings schon in früheren Fällen von zentraler Bedeutung für Radikalisierungen. Für die Mitglieder der DTM und ihr Unterstützerumfeld (in Deutschland) war das Internet ein wichtiges Werkzeug, um neue Anhänger zu mobilisieren und die Radikalisierung unter ihren Mitgliedern zu forcieren. Im Folgenden soll daher anhand des Falles von Fatma, die unter anderem für die DTM Propaganda im Internet betrieben hat, aufgezeigt werden, wie sich Radikalisierungen durch Kontakte und Interaktionen im Internet vollziehen können.

\section{Einzelfallanalyse von Fatma}

Im März 2011 wurde eine deutsche Jihadistin am Berliner Kammergericht wegen der Werbung für und Unterstützung von terroristische(n) Vereinigungen zu zwei Jahren und sechs Monaten Haft verurteilt. Wie oben bereits erwähnt wird die Jihadistin hier Fatma genannt, da aus datenrechtlichen Gründen ihr bürgerlicher Name nicht 
angegeben werden darf. Fatma hatte von 2009 bis 2010 Werbung für terroristische Vereinigungen wie Al-Qaida und die IJU im Internet betrieben. Durch ihre Propagandatätigkeit im Internet kam sie mit anderen Jihadist*innen in Kontakt, die in afghanisch-pakistanischen Konfliktgebieten als Mitglieder einer terroristischen Vereinigung aktiv waren. Diese Internetkontakte beförderten ihren Radikalisierungsprozess nachdrücklich. Sie hielt sich täglich in extremistischen, virtuellen Gemeinschaften auf, stand dort mit zahlreichen Jihadist*innen aus dem In- und Ausland in Kontakt und kommunizierte mit ihnen. Diese Interaktionen wirkten sich radikalisierend auf ihre weitere Entwicklung aus. Erst durch die Kontakte im Internet wurde Fatma eine zentrale Unterstützerin der DTM. Ihr Fall veranschaulicht, dass Radikalisierungen nicht nur durch Face-to-Face-Kontakte, sondern auch durch Konsum jihadistischer Inhalte im Internet sowie Interaktionen mit jihadistischen Aktivist*innen in der virtuellen Welt ausgelöst und intensiviert werden können.

Vor der Analyse des Radikalisierungsprozesses von Fatma und die Rolle des Internets in diesem Prozess erfolgt im Folgenden eine kurze Darstellung des Sozialisationshintergrunds von Fatma.

\subsection{Biografische Angaben}

Fatma wurde in den frühen 1980er-Jahren in Memmingen geboren und lebte bis Mitte der 1980er-Jahre mit ihren kurdischen Eltern dort. Nach der Scheidung ihrer Eltern lebte Fatma mit ihrer Mutter und dem jüngeren Bruder in Ulm. Dort besuchte sie die Grundschule und später die Hauptschule, die sie abschloss. Mit 16 Jahren nahm sie die deutsche Staatsbürgerschaft an. In diesem Zeitraum fing sie eine zweijährige Ausbildung zur Einzelhandelskauffrau in einem Ulmer Schuhgeschäft an, die sie im Sommer 2000 erfolgreich abschloss. Die Inhaber des Schuhgeschäfts stellten sie allerdings nur für einen weiteren Monat ein. Seitdem arbeitete Fatma in zahlreichen befristeten Arbeitsverhältnissen, meistens im Bereich des telefonischen Kundenservice (Gerichtsverfahren gegen Fatma vom 9. März 2011).

Fatma kommt aus einer kurdischstämmigen Familie, die ursprünglich nicht religiös war. Ihre Familie besuchte nur selten die Moschee und befolgte zumeist keine religiösen Glaubensgebote. Von ihrer Mutter wurde sie streng, aber nicht religiös erzogen. Die Mutter scheint gewalttätig gegenüber ihrer Tochter gewesen zu sein. Erst Mitte der 2000er Jahre entwickelten Fatmas Mutter und einer ihrer Onkel ein gewisses Interesse für den Islam. Beide führten den Hadsch durch, die Pilgerfahrt nach Mekka. Nach der Reise erzählte die Mutter viel von ihren Eindrücken auf der Pilgerfahrt. Fatma scheint von den Erzählungen ihrer Mutter stark beeindruckt gewesen zu sein. Schon wenige Monate später begann sie fünfmal am Tag zu beten und sich immer häufiger an sehr rigide Glaubensvorschriften zu halten. Seit 2006 besuchte sie auch Moscheen in Ulm und Umgebung. Da sie zu diesem Zeitpunkt immer mehr ihr äußeres Erscheinungsbild änderte, kann davon ausgegangen werden, dass sie sich schon damals regelmäßig in der Neu-Ulmer Moschee des Multikulturhauses aufhielt. Das Multikulturhaus war damals einer der radikalsten Treffpunkte der islamistischen Szene in Deutschland, weil dort ranghohe Jihadisten wie Yahia Yussuf, Adly el-Attar und Reda Seyam ihre Missionsarbeit betrieben (Ramelsberger 2008, S. 92-110). 
Im Oktober 2006 lernte sie den Konvertiten G. im Multikulturhaus kennen. G. geriet durch einen Schulfreund, Tolga D., in das radikale Milieu des Multikulturhauses. Dort freundete er sich mit einer kleinen Gruppe junger Muslime an, die regelmäßig die Islamseminare des ägyptischen Predigers Yahia Yussuf besuchten. Unter dem Einfluss des Predigers entwickelten die Mitglieder der Gruppe ein immer größer werdendes Interesse daran, sich einer jihadistischen Bewegung im Ausland anzuschließen (Steinberg 2014, S. 95-97). Im Juni 2006 schaffte es G. zusammen mit Adem Y., den er 2005 in Mekka kennengelernt hatte, nach Waziristan in Pakistan zu reisen. Dort erhielten beide eine militärische Ausbildung in einem Trainingscamp der IJU, einer terroristischen Vereinigung, deren Mitglieder aus Usbekistan stammten (Steinberg 2014, S. 88-112).

Fatma traf G. wenige Monate nach seiner Rückkehr aus Pakistan. Nach kurzer Zeit entschlossen sich die beiden zu heiraten. Obwohl Fatmas Mutter gegen eine Hochzeit mit einem Konvertiten war, heirateten sie im Januar 2007 und zogen in eine gemeinsame Wohnung in Neu-Ulm (Gerichtsverfahren gegen Fatma vom 9. März 2011). Am 4. September 2007 verhaftete das Bundeskriminalamt G. und zwei weitere Jihadisten in einem kleinen Dorf im Sauerland. Die drei Jihadisten der sogenannten Sauerland-Gruppe wurden von der Polizei verdächtigt, einen oder mehrere Terroranschläge geplant und vorbereitet und die hierfür vorgesehenen Bomben gebaut zu haben. Zwei Monate später verhaftete die Polizei noch eine vierte Person in der Türkei, der eine Beteiligung an der Anschlagsplanung vorgeworfen wurde.

Fatma gab in ihrem eigenen Gerichtsverfahren im Jahr 2011 an, dass sie nichts von den Anschlagsplänen ihres Ehemanns gewusst und erst nach dessen Verhaftung hiervon erfahren habe. Seine Festnahme habe bei ihr einen Schockzustand ausgelöst. Vor Gericht sagte sie aus: „Ich habe neben mir gestanden.“ (Gerichtsverfahren gegen Fatma vom 1. März 2011) Außerdem sei sie in der Folge sozial isoliert gewesen, weil sie im Freundeskreis aufgrund der terroristischen Gesinnung ihres Ehemanns heftig kritisiert worden war und man sich von ihr abgewandt hatte. Im Gerichtsverfahren erzählte sie, sie habe damals ,nur noch Allah auf ihrer Seite“ gehabt (Gerichtsverfahren gegen Fatma vom 1. März 2011). Da sie nach der Festnahme ihres Mannes zu ihrer Mutter zurückziehen musste, brachen alte familiäre Konflikte wieder auf. Die Spannungen zwischen Mutter und Tochter beruhten nicht zuletzt darauf, dass die Mutter ihre Hochzeit mit G. missbilligte und sich nun durch dessen Festnahme in ihrer Haltung bestätigt sah. Sie forderte ihre Tochter auf, sich scheiden zu lassen, was diese jedoch ablehnte. Fatma geriet somit durch den Umzug erneut unter die strenge Aufsicht ihrer Mutter, was sie als belastend und demütigend empfand. So sagte sie vor Gericht: „Ich habe durch die Festnahme die Freiheit verloren, weil ich zurück zu meiner Familie musste." (Gerichtsverfahren gegen Fatma vom 1. März 2011)

\subsection{Der Radikalisierungsprozess von Fatma im Internet}

Fatma suchte im Internet nach neuen sozialen Kontakten, um ihre soziale Isolation zu kompensieren. Dabei versuchte sie auch Kontakt zu Jihadist*innen aufzunehmen. So nutzte sie beispielsweise die Videoplattform YouTube, um mit deutschen Jihadist*innen, die dort einen Videokanal besaßen, in Kontakt zu kommen und mit 
ihnen zu kommunizieren. Fatma wies am Kammergericht darauf hin, dass sie sich Informationen von den jihadistischen Internetaktivist*innen erhoffte, weswegen ihr Mann verhaftet worden sei und wie es zu seiner Verhaftung kommen konnte (Gerichtsverfahren gegen Fatma vom 22. November 2010).

Ihre Angaben in dem Geständnis veranschaulichen, dass Fatma der medialen Berichterstattung über die Festnahmen der so genannten Sauerland-Gruppe misstraute. Für sie waren die Hinweise, die ihr die jihadistischen Internetaktivist*innen über ihren Mann und dessen Aktivitäten gaben, glaubwürdiger als die Berichte aus dem Fernsehen und den Zeitungen. Dementsprechend gab sie auch in ihrem Geständnis vor dem Berliner Kammergericht an, dass sie Informationen über die Festnahme ihres Mannes von ihren Brüdern erhalten wollte (Gerichtsverfahren vom 22. Dezember 2010). Sie suchte bewusst im Internet nach Gleichgesinnten, die ihr über die Ereignisse der Festnahme aus einer muslimischen Perspektive berichten könnten. Allerdings vermittelten die Jihadist*innen der jungen Frau eine ihrem Deutungsrahmen entsprechende Version der Geschehnisse, wonach es sich bei G.s Festnahme um eine anti-islamische Handlung von Seiten der staatlichen Sicherheitsbehörden handelte.

Anhand Fatmas Aussagen vor dem Kammergericht ist ersichtlich, dass sie seit Ende 2007 regelmäßig jihadistische Internetseiten besuchte. In der Anfangsphase nutzte sie vor allem die Videoplattform YouTube, auf der auch ihr erster eigener Videokanal unter dem Pseudonym fisebilillah eingerichtet war. Fisebilillah heißt übersetzt: auf dem Weg Allahs. Der Vorsitzende Richter am Berliner Kammergericht fragte Fatma, wieso sie diesen Begriff im Internet verwendet habe. Fatma argumentierte, sie habe den Begriff gewählt, weil sie in ihrem Freundeskreis wegen ihrer radikalen Einstellungen kritisiert worden sei und dadurch gesellschaftlich immer mehr isoliert gewesen sei. Nur noch Allah habe an ihrer Seite gestanden. Daher sei sie gezwungen gewesen, sich im Internet einen neuen Freundeskreis zu erschließen. Da dieser virtuelle Freundeskreis ebenfalls dem Weg Allahs gefolgt seie, hätte sie sich ihm im Internet angeschlossen (Gerichtsverfahren vom 20. November 2010).

Über das Internet erhielt Fatma auch Informationen über den Krieg in Afghanistan. Da ihr jihadistische Internetaktivist*innen über die für die dort kämpfenden Jihadist*innen schlechte Situation in den Kriegsgebieten berichteten, beschäftigte sie sich immer mehr mit der Thematik der westlichen Militäreinsätze in der islamischen Welt.

Ihre Informationen erhielt sie nicht nur über jihadistische Videokanäle, sondern sie besuchte auch deutsch-, englisch- und türkischsprachige Webforen, die salafistische oder jihadistische Internetaktivist*innen betrieben. So meldete sich Fatma 2008 bei dem salafistischen Webforum Ahlu-Sunnah ${ }^{2}$ an. Das Forum, dessen Betreiber laut Hamburger Verfassungsschutzbericht von 2011 zum Teil in Hamburg lebten, wurde 2008 gegründet und galt seit Anfang der 2010er Jahre als das führende deutschsprachige Forum, in dem salafistische Positionen zu verschiedenen Themen diskutiert und verbreitet wurden (Landesamt für Verfassungsschutz Hamburg 2012, S. 42).

Die Mitglieder des Forums befassten sich aber nicht nur mit salafistischen Fragen, sondern es wurden auch jihadistische Beiträge im Forum veröffentlicht. Fatma nutzte

2 Die Domain des Forums war http://ahlu-sunnah.com/index.php. 
im Ahlu-Sunnah-Forum anfangs das Pseudonym Schokocafe. Da sie in Diskussionen mit anderen Teilnehmer*innen äußerst radikale Positionen vertrat, wurde sie mehrfach im Forum gesperrt und musste sich dann (unter einem anderen Namen) neu anmelden (Gerichtsverfahren gegen Fatma vom 22. Dezember 2010). Außer Fatma agierten auch zahlreiche andere jihadistische Internetaktivist*innen in dem salafistischen Forum, was immer wieder zu Konflikten mit anderen Teilnehmer*innen führte, weil sie diese provozierten oder diffamierten. Auf Druck der Sicherheitsbehörden musste das Webforum 2014 schließlich geschlossen werden.

Neben dem Ahlu-Sunnah-Forum war Fatma auch in dem türkischsprachigen Takva-Forum aktiv, das von Herbst 2007 bis Sommer 2009 im Internet existierte und in dem Propagandamaterial zahlreicher jihadistischer Bewegungen, das aus dem Arabischen ins Türkische und/oder Englische übersetzt wurde, verbreitet wurde. ${ }^{3}$ Die türkischen Betreiber*innen waren Anhänger von Al-Qaida. Dies war zum einen daraus ersichtlich, dass auf der Webseite Bilder von Osama bin Laden, Ayman alZawahiri und Abu Yahya Libi zu sehen waren. Zum anderen übersetzten die Internetaktivist*innen aber auch fast alle Botschaften und Videos des Al-Qaida-Medienarms as Sahab ins Türkische. Fatma veröffentlichte in dem Forum nur türkischsprachige Beiträge.

Im März 2009 registrierte sie sich dann im englisch- und im deutschsprachigen Forum des Ansar-Netzwerkes, in Letzterem wiederum unter dem Pseudonym fisebilillah. Das Ansar-Netzwerk umfasste insgesamt drei jihadistische Webforen, die in drei verschiedenen Sprachen jihadistisches Propagandamaterial im Internet verbreiteten. Das erste Forum, das von dem in Spanien lebenden Marokkaner Faisal Errai unter dem Decknamen Abu Hafes al-Maghrebi geleitet wurde, war arabischsprachig und existierte seit Frühjahr 2008. In dem Forum wurden Texte, Bücher und Videobotschaften jihadistischer Bewegungen veröffentlicht. In der Anfangsphase nutzten nur ca. 400 Mitglieder dieses Forum. Innerhalb von drei Jahren stieg die Zahl der registrierten Mitglieder aber auf 8000 Nutzer an (Torres-Soriano 2013, S. 169). Dadurch wurde das Ansar-Netzwerk zwischen 2009 und 2012 zu einer der wichtigsten jihadistischen Propaganda-Plattformen im Internet. Errai war der Chef der Medienabteilung des Ansar-Netzwerkes, weil er neben dem arabischen Forum auch maßgeblich Einfluss auf die Etablierung der englisch- und deutschsprachigen Webforen hatte. Im Frühjahr 2009 ging das deutschsprachige Forum online, ${ }^{4}$ das der im rheinland-pfälzischen Montabaur lebende staatenlose Palästinenser Hussam S. mit Errais Hilfe aufgebaut hatte (Gerichtsverfahren gegen Hussam S. vom 8. November 2011).

Aufgrund ihrer Verbindung mit einem verurteilten jihadistischen Terroristen erhielt Fatma von S., der auch der Administrator des deutschsprachigen Forums war, den Status einer sogenannten Supermoderatorin. In dieser Funktion konnte Fatma sowohl jihadistisches Propagandamaterial der IJU und DTM im Forum veröffentlichen als auch eigene Texte und Beiträge publizieren. In einem Zeitraum von gut zehn Monaten veröffentlichte sie fast 1000 Kommentare und Beiträge. Zudem hatte sie als Supermoderatorin die Möglichkeit missliebige Beiträge von anderen Mitglie-

\footnotetext{
3 Die Domain des Forums war http://forum.tak-va.com/index.php.

4 Die Domain des Forums war http://ansar1.info.
} 
dern des Forums zu entfernen (Gerichtsverfahren gegen Fatma vom 20. November 2010).

Im deutschsprachigen Ansar al Jihad-Forum sprach sich unter den Mitgliedern schnell herum, dass hinter dem Pseudonym fisebilillah die Ehefrau von G. steckte. Durch ihren Sonderstatus lernte sie im Forum zahlreiche jihadistische Aktivist*innen und Sympathisant*innen kennen. Unter ihnen befand sich auch der in Salzgitter geborene Türke Ahmed Manavbasi, der von 2009 bis 2010 der sogenannte Emir der Deutschen Taliban Mudschahideen war. Im Forum nutze er das Pseudonym Selehaddin. Manavbasi lebte schon einige Jahre in Waziristan/Pakistan (Gerichtverfahren gegen Thomas U. vom 14. November 2012). ${ }^{5}$ Er hatte sich dort der IJU angeschlossen und leitete von April 2007 bis Januar 2009 die Medienarbeit dieser ursprünglich aus Usbekistan stammenden terroristischen Gruppe (Gerichtverfahren gegen Fatma vom 9. Februar 2011). Im deutschsprachigen Ansar-al-Jihad-Forum veröffentlichte er seine für die IJU produzierten Video- und Textbotschaften. Im Laufe der Zeit entwickelte sich zwischen Manavbasi und Fatma eine engere virtuelle Beziehung. So kommunizierten sie fast täglich über Chatforen und E-Mails. Sie unterhielten sich in ihren Chats über allgemeine Themen wie die aktuelle Situation und die Lebensverhältnisse in den pakistanischen Stammesgebieten. Manavbasi gab ihr gegenüber Auskunft über sein derzeitiges Befinden. Aufgrund des US-Drohnenkrieges war die Situation der in Waziristan lebenden Menschen ziemlich angespannt. Zugleich gingen beide in ihren Chats auch auf private Themen ein. So erzählte Fatma Manavbasi zum Beispiel über ihre Probleme mit ihrer Mutter und fragte ihn, ob es erlaubt sei, als Frau alleine zu leben (Gerichtsverfahren gegen Fatma vom 20. Januar 2011).

Nach wenigen Wochen versuchte Manavbasi die Deutsch-Kurdin immer mehr in die Arbeit der Medienproduktion von Elif Medya einzubinden. Manavbasi hatte am 12. Februar 2009 eine eigene Medienproduktion unter den Namen Elif Medya gegründet, um unabhängig von der IJU gezielt Propaganda für ein deutschsprachiges Publikum zu machen. Im Internet kündigte er die Gründung mit der Etablierung eines Blogs (elifmedya.wordpress.com) an (Gerichtsverfahren gegen Fatma vom 20. Januar 2011). Unter dem Logo von Elif Medya produzierte Manavbasi Videos und Texte für die DTM und Tafetül Mansura (Steinberg 2012). ${ }^{6}$ Anfangs verbreitete die Videos und Texte eine Kontaktperson von Manavbasi aus Istanbul. Er veröffentlichte die Beiträge der DTM auf der türkischen Webseite www.sehatzamani.com. Um größere Aufmerksamkeit zu erhalten, forderte Manavbasi Fatma dazu auf, die Video- oder Textbeiträge von Elif Medya auch auf Videokanälen und in Webforen zu veröffentlichen. Fatma gründete im Frühjahr 2009 bis zu neun Videokanäle auf YouTube, um unter anderem die Videos von Elif Medya zu verbreiten. Ihre Videokanäle liefen unter den bereits erwähnten Namen fisebilillah und Schokocafe, des Weiteren unter Umm Asadullah, xUmmSayfullah', XamatullahX, muadha, tavheed, SUNNAinDaHouse und tawhed (Gerichtverfahren gegen Fatma vom 26. Januar 2011). Auf diesen Kanälen veröffentlichte sie über 100 Videos. 30 Videos waren Kurzfilme mit Texten, die sie selbst geschrieben hatte (Gerichtverfahren gegen Fatma vom 26.

\footnotetext{
5 Aussage eines Sachverständigen des BKA im Gerichtverfahren gegen Thomas U.

6 Steinberg bezeichnet die Gruppe Tafetül Mansura als türkisch-aserbaidschanische Schwesterorganisation der Deutschen Taliban Mudschahideen.
} 
November 2010). Die restlichen Videos waren entweder Medienproduktionen von Elif Medya, die Manavbasi für die IJU oder die Deutschen Taliban Mudschahideen produziert hatte oder es handelte sich um Produktionen von as Sahab (Al-Qaida). So veröffentlichte Fatma auf dem Videokanal badr at tawhed türkischsprachige Videos, die Manavbasi für die IJU angefertigt hatte. Im Ansar-al-Jihad-Forum verbreitete sie das erste Video der DTM mit dem Titel „Der Ruf zur Wahrheit“ (Gerichtverfahren gegen Fatma vom 26. Januar 2011). Mit dem Video versuchten die deutschen Jihadisten um Eric Breininger, Yusuf Ocak und Fatih Temelli neue Gleichgesinnte aus Deutschland für den bewaffneten Kampf im afghanisch-pakistanischen Grenzgebiet zu mobilisieren. Am 24. Januar 2010 verbreitete Fatma einen weiteren Beitrag der DTM mit dem Titel „Der einzige Weg ist der Widerstand und die Verbundenheit mit dem Jihad“. Mit diesen Veröffentlichungen im Internet konnten die Richter am Berliner Kammergericht Fatma nachweisen, dass sie gezielt terroristische Gruppen im Ausland mit ihren propagandistischen Internetkampagnen unterstützte.

Daneben wurde Fatma auch in die Produktion des Medienarms Elif Medya eingebunden. Manavbasi instruierte sie, eigene Beiträge zu produzieren. So forderte er sie beispielsweise im Februar 2010 in einem Internetchat auf, einen Beitrag über das Burka-Verbot in Frankreich und in anderen europäischen Ländern zu erstellen (Gerichtverfahren gegen Fatma vom 7. Januar 2011). Bei seinen Instruktionen setzte Manavbasi die damals 28-jährige Fatma immer wieder unter Druck, indem er auf die katastrophalen Bedingungen in Waziristan verwies. So schrieb er ihr in einer E-Mail: „Wieso kommen keine Mudschahideen nach Waziristan, obwohl es so schlimm ist?“ (Gerichtverfahren gegen Fatma vom 7. Januar 2011). Mit dieser Frage forderte er Fatma auf, sich mehr für die DTM zu engagieren und deutsche Jihadist*innen für den Kampf in Waziristan zu rekrutieren. Er setzte Fatma moralisch unter Druck, um bei ihr Gewissenskonflikte herbeizuführen, damit sie sich gezwungen sieht, der DTM zu helfen.

Neben der gezielten Werbung für die terroristischen Gruppen im Internet verbreitete sie allerdings auch Videos, die sie selber produziert hatte. Diese Videos waren bei weitem nicht so professional produziert wie die Video- und Textbotschaften, die Ahmed Manavbasi für Elif Medya produziert hatte. So veröffentlichte Fatma auf dem Kanal tawhed beispielsweise ein Video mit dem Titel „Unsere Löwen brauchen uns", welches vorrangig aus wenigen Bildern und einem kurzen Text besteht. In der Videobotschaft werden Frauen dazu aufgefordert, ihre Männer - die Löwen - im Jihad und bei der Vernichtung der Feinde des Islams zu unterstützen (Gerichtsverfahren gegen Fatma vom 20. November 2010). In einem weiteren Video mit dem Titel „Traurig aber wahr ...“, der auf dem Kanal XamatullahX veröffentlicht wurde, beschäftigte sich Fatma mit dem Thema westlicher Militäreinsätze in muslimischen Ländern. In dem Video sind Filmsequenzen zu sehen, in denen das Leiden der Muslim*innen in Kriegsgebieten gezeigt wird. Als ihr nach der Veröffentlichung dieses Videos vorgeworfen wurde, dass sie mit Unterstellungen arbeiten würde, erwiderte sie, dass es für alle Aussagen in dem Video zahlreiche Beweise gebe. Auch müsse man nur die kritische Lage betrachten, in der sich die Ummah (Gemeinschaft aller Muslim*innen) befinde, um festzustellen, dass Muslim*innen schlecht und ungerecht behandelt würden. Anhand dieser Beispiele lässt sich sehr anschaulich zeigen, dass Fatma die wichtigsten jihadistischen Narrative stark verinnerlicht hatte 
und dem entsprechend auch in ihren selbst produzierten Videos einsetzte. Mit ihren Propagandabotschaften versuchte sie nicht nur Männer für den bewaffneten Kampf in Waziristan zu rekrutieren, sondern auch Frauen als Unterstützerinnen zu mobilisieren. So thematisierte sie auch in einigen selbst produzierten Videos die Rolle der Frau im bewaffneten Kampf gegen die Ungläubigen (Gerichtverfahren vom 26. Januar 2011).

Unter den selbst produzierten Beiträgen verbreitete Fatma auch Videobotschaften, in denen Bilder oder Filmausschnitte von toten oder verletzten Kindern sowie weinenden Frauen zu sehen waren. Auf ihrem YouTube-Kanal SUNNAinDAhouse präsentierte sie beispielsweise das selbst produzierte Kurzvideo „Das Mindeste was wir für unsere Ummah tun können ...“, in dem man mehrere Bilder von verletzten oder getöteten Kindern sieht, die von ihren Vätern bzw. von Männern im Arm getragen werden. Als Erstes zielte sie mit diesem visuellen Framing darauf, ihrer Zielgruppe zu verdeutlichen, dass unschuldige Kinder Opfer westlicher Militäroperationen sind. Auf diese Weise vermittelte sie den Betrachtern das Bild, dass nicht nur muslimische Erwachsene, sondern auch Kinder Opfer eines Aggressors sind, der jegliche Achtung vor dem menschlichen Leben verloren hat. Damit wollte sie den Beweis erbringen, dass westliche Soldat*innen nicht vor Verbrechen gegen Kinder zurückschrecken. Zugleich versuchte sie einen Prozess der Dehumanisierung zu erzeugen, der dazu beitragen sollte, dass bei den Betrachtenden die Hemmschwelle zur Gewaltanwendung sinkt. Fatma beabsichtigte mit ihrem image warfare (Roger 2013) eine emotionale sowie kognitive Distanz zwischen Publikum und dem Aggressor bzw. der anzugreifenden Zielgruppe herzustellen, um die psychologischen Barrieren zur Anwendung von politischer Gewalt oder Terror zu beseitigen (Borum 2011, S. 29; Bötticher und Mares 2012, S. 188-189).

Dabei ergänzte Fatma die Kriegsbilder mit einem Text, in dem sie die Untätigkeit deutscher Muslim*innen anprangerte. Obwohl muslimischen Kindern in den Kriegsgebieten tagtäglich Unrecht widerfahre, würden die Muslim*innen in Deutschland den Opfern nicht zu Hilfe kommen.

Obwohl die ganze Ummah, ganz gleich welcher Nationalität, Rasse und Hautfarbe sie angehören, sich gegen diese unmenschlichen Handlungen widersetzen muss, begnügt sie sich leider nur damit, den Grausamkeiten zuzusehen. Denkt ihr etwa, dass es reicht, wenn wir uns die Bilder anschauen, wütend werden und Mitleid haben? [...] Erfüllen wir unsere Verantwortung gegenüber unseren Glaubensbrüdern und -schwestern, die täglich [...] Folterungen und Misshandlungen erleben? [...] Meine lieben Geschwister, die Ummah schreit nach uns ... [...] Ein Muslim, der behauptet, dass er glaubt, kann nicht dem Leiden seiner Geschwister zusehen und dabei regungslos bleiben. (SUNNAinDAhouse 2009)

Der Text fungierte als mobilisierender Deutungsrahmen, der bei den Betrachter*innen Anreize zum (kollektiven) Handeln schaffen sollte. Um diesen Effekt zu verstärken, verfasste Fatma den Schuldappell, der beim Publikum ein schlechtes Gewissen hervorrufen sollte. So warf sie explizit ihren muslimischen Zuschauer*innen vor, im Westen ein Leben in Sicherheit und Wohlstand zu führen, aber nicht den Kriegsopfern in den muslimischen Ländern helfen zu wollen. 
Dass solche Schuldappelle bei muslimischen Jugendlichen Einstellungs- und Verhaltensänderungen hervorrufen, ist beispielsweise durch die Aussagen des kanadischen Jihadisten Momin Khawaja bekannt, der 2004 in Ottawa verhaftet wurde, weil er eine britische Terrorzelle unterstützte (Schwartz 2012). In einer E-Mail schrieb er, dass er in relativem Luxus und Sicherheit leben würde, ,whilst others were living lives of oppression and fear. He was healthy and well fed, while others were dying or leading lives of deprivation" (Khawaja, zit. n. Quiggin 2010, S. 87). Nachdem Khawaja Filmausschnitte und Videos von der palästinensischen Intifada und dem Krieg in Tschetschenien gesehen habe, sei für ihn deutlich geworden, dass es einen signifikanten Unterschied zwischen seinem Leben und dem vieler anderer Muslim*innen in der Welt gäbe. Er sei ein Muslim, der im Gegensatz zu vielen anderen Muslim*innen, in einem Land leben würde, das für das Leiden der Muslim*innen in der Welt mitverantwortlich sei. Diese Erkenntnis führte bei ihm zu dem Entschluss, sein Leben zu ändern (Quiggin 2010, S. 87-88).

Nicht nur Khawaja, sondern zahlreiche junge Muslim*innen, die im Westen leben, sehen sich gezwungen, ihre Lebensweise grundlegend zu verändern, wenn ihnen bewusst wird, unter welchen Bedingungen Muslim*innen in Konflikt- und Kriegsgebieten leben. Sie haben ein schlechtes Gewissen, weil sie selber ein gutes Leben führen. Deswegen sehen sie sich veranlasst, den Opfern in den Kriegsgebieten zu helfen, indem sie die dort kämpfenden, jihadistischen Bewegungen unterstützen (Anzalone 2012; Mastors und Siers 2014).

Auf diesen Impuls setze auch Fatma als jihadistische Internetaktivistin. Mit den Kriegsbildern versuchte sie kollektive Handlungsimpulse zu befördern. Da hinter den Bildern reale Ereignisse stecken, sollten die Betrachter*innen motiviert werden, gegen das Unrecht vorzugehen. So wies beispielsweise Fatma in ihrem Video „Das Mindeste was wir für unsere Ummah tun können ...“ auf die Dringlichkeit zum Handeln hin, in dem sie dem Publikum verdeutlichte, wenn sie nicht am Kampf gegen die Ungläubigen teilnehmen würden, noch mehr Frauen und Kinder sterben müssten. Somit dramatisierte Fatma bewusst die Lage der muslimischen Bevölkerung in den Kriegsgebieten, um eine möglichst große Zahl der jungen Betrachter*innen dazu zu motivieren, in den Jihad nach Afghanistan oder Pakistan zu ziehen. Gezielt nutzte sie in ihren Videos das motivierende Framing, um einen hohen moralischen Druck auf die Betrachter*innen auszuüben und sie dadurch für ihre politischen Zwecke zu mobilisieren.

\section{Fazit}

In der Einzelfallanalyse konnte festgestellt werden, dass Fatma durch ihre (Propaganda-)Aktivitäten in den Sozialen Medien als radicalizing agent (Bouhana und Wikström 2011, S. 37) fungierte. Von 2008 bis 2010 war sie eine Schlüsselperson der virtuellen Welt des Jihadismus in Deutschland, die maßgeblich zur Verbreitung deutschsprachiger jihadistischer Text- und Videobotschaften im Internet beigetragen hatte. Ihre virtuellen Kontakte zu Jihadist*innen im Ausland führten dazu, dass sie durch ihre Unterstützungsarbeiten ein offizielles Mitglied des Medienarms der 
Deutschen Taliban Mudschahideen (Elif Medya) wurde. Sie war maßgeblich an der Entstehung der Internetpräsenz einer terroristischen Vereinigung beteiligt.

Anhand ihrer Internetaktivitäten wurde deutlich aufgezeigt, dass es Propagandist*innen gelingt, im Internet und den Sozialen Medien Jugendliche mit jihadistischen Botschaften zu erreichen und zu beeinflussen. Zusätzlich war sie, durch ihre virtuellen Kontakte zu deutschen und türkischen Jihadist*innen in Pakistan, in der Lage, Jugendliche aus Deutschland für jihadistische Vereinigungen zu mobilisieren. Durch die Einzelfallanalyse von Fatmas Radikalisierungsprozess ist es möglich, ein Modell von Radikalisierungen vorzuschlagen, welches den Einfluss des Internets bzw. der Sozialen Medien bei Radikalisierungen aufzeigt. Mit dem Modell wird dargelegt, welche Stufen eine Person im Radikalisierungsprozess, beeinflusst durch das Internet und die Sozialen Medien, durchlaufen kann. Die Radikalisierung im hier vorgestellten Fall besteht aus fünf Stufen:

Durch die Auswertung der Daten aus dem Gerichtsverfahren gegen Fatma wurde bekannt, dass sie seit spätestens Ende 2007 regelmäßig das Internet und Soziale Medien nutzte. Nach der Verhaftung ihres Mannes fühlte sie sich durch die mediale Berichterstattung sozial isoliert. Viele Freund*innen meldeten sich nicht mehr bei ihr oder distanzierten sich von ihr. Da sie kaum noch Freund*innen hatte, mit denen sie reden konnte, suchte sie neue Bezugspersonen im Internet. In der ersten Phase ihrer Online-Radikalisierung, die hier Suchphase genannt wird, spielt das Alleinsein und die Isolierung von ihrem bisherigen sozialen Umfeld eine bedeutende Rolle. Fatma lernte im Internet Personen kennen, die dem jihadistischen Milieu nahestanden. In den ersten Jahren (2007-2008) nutzte sie hauptsächlich die Videoplattform YouTube, um mit anderen Personen in Kontakt zu kommen und mit ihnen zu kommunizieren. Ab Mitte 2008 begann sie auch zahlreiche salafistische und jihadistische Webforen zu nutzen, um in Kontakt mit Gleichgesinnten zu kommen. Durch ihre Internetaktivitäten konnte sich Fatma einen neuen virtuellen Freundeskreis aufbauen und sich mit ihren neuen Freund*innen immer mehr vernetzen. Die zweite Phase kann aus diesem Grund als Vernetzungsphase angesehen werden. Durch die stetig wachsenden, virtuellen Kontakte gelangte Fatma in der dritten Phase immer tiefer in die virtuelle Gemeinschaft des deutschen jihadistischen Milieus. Sie wurde dadurch ein aktives Mitglied dieser virtuellen Gemeinschaft, in der u. a. auch (ideologische) Diskussionen geführt wurden. Solche Diskussionen dienten dazu, jihadistische Deutungsrahmen zu verinnerlichen, Feindbilder aufzubauen und dabei ihre Feinde zu dehumanisieren. Die dritte Phase gilt als Bestätigungs- oder Überzeugungsphase, da sich Sympathisant*innen wie Fatma in den Gesprächen in den Sozialen Medien immer mehr mit jihadistischen Deutungsmustern auseinandersetzen, sich mit ihren virtuellen Freund*innen gegenseitig in ihren Positionen bestärken und dadurch immer radikalere Ansichten erhalten (Effekt der Echokammer). Zugleich hilft jungen Männern und Frauen die Anbindung an und Kommunikation in virtuelle(n) Netzwerke(n), um Bestätigung von anderen, gleichgesinnten Personen zu erhalten und dadurch ein gewisses Zusammengehörigkeitsgefühl zu entwickeln. Schließlich kommen in der darauffolgenden - vierten - Kooperationsphase Sympathisant*innen bzw. Anhänger*innen in immer engeren Kontakt mit jihadistischen Propagandisten oder Terrorist*innen, die zumeist Mitglieder terroristischer Vereinigungen im Ausland sind. Solche virtuellen Kontakte erfolgten in den Jahren 2006 bis 2012 häufig 
über jihadistische Webforen, in denen auch jihadistische Propagandist*innen und Terrorist*innen aus dem Ausland agierten, um neue potenzielle Anhänger*innen für ihre terroristischen Vereinigungen zu rekrutieren. So lernte Fatma beispielsweise den in Salzgitter geborenen Türken Ahmed Manavbasi über das Ansar al-Jihad-Forum kennen. Manavbasi hatte sich 2006 der terroristischen Vereinigung IJU in Pakistan angeschlossen und war zwischen 2009 und 2010 die zentrale Führungsfigur der jihadistischen Gruppe der DTM. Die stark zunehmenden, virtuellen Interaktionen führten letztendlich dazu, dass Fatma immer mehr in die Propagandaarbeit der DTM eingebunden und involviert wurde. Fatma half den Mitgliedern der DTM bei der Verbreitung extremistischer Botschaften im Internet. Folglich kann die letzte Phase als Operations- und Unterstützungsphase bezeichnet werden. Denn Fatma übernahm dabei eine bedeutend aktivere Rolle in der Propaganda, insbesondere indem sie für die DTM Videos und anderen Medienbeiträgen produzierte, die Internetseiten der DTM verwaltete und einen Großteil des Propagandamaterials im Internet verbreitete.

Das hier vorgestellte Fünf-Stufen-Modell verdeutlicht, dass das Internet und die Sozialen Medien eine zentrale Rolle bei dem Radikalisierungsprozess von Fatma spielten. Fatma nutzte das Internet sehr intensiv und wurde dadurch immer mehr in die virtuellen Gemeinschaften jihadistischer Netzwerke integriert. Die Kontakte und Interaktionen in den virtuellen Gemeinschaften beschleunigten und verstärkten ihren Radikalisierungsprozess, weil sie sich fast jeden Tag in den Sozialen Medien aufhielt, dort mit jihadistischen Aktivist*innen bzw. Terrorist*innen kommunizierte und diese dadurch einen immer größeren Einfluss auf ihre Einstellungen und Verhaltensweisen gewannen. Dabei gelang es insbesondere den Akteur*innen der DTM, Fatma von einer Sympathisantin zu einer aktiven Propagandistin und Unterstützerin einer terroristischen Vereinigung im Ausland zu machen.

\section{Gerichtsverfahren}

- Verhandlung vom 20. November 2010 gegen Fatma am Kammergericht in Berlin.

- Verhandlung vom 22. Dezember 2010 gegen Fatma am Kammergericht in Berlin.

- Verhandlung vom 7. Januar 2011 gegen Fatma am Kammergericht in Berlin.

- Verhandlung vom 26. Januar 2011 gegen Fatma am Kammergericht in Berlin.

- Verhandlung vom 9. Februar 2011 gegen Fatma am Kammergericht in Berlin.

- Verhandlung vom 1. März 2011 gegen Fatma am Kammergericht in Berlin.

- Verhandlung vom 9. März 2011 gegen Fatma am Kammergericht in Berlin.

- Verhandlung vom 14. November 2012 gegen Thomas U. am Kammergericht in Berlin.

- Verhandlung vom 3. April 2012 gegen Fatih K. am Kammergericht in Berlin.

- Verhandlung vom 8. November 2011 gegen Hussam S. am Oberlandesgericht Koblenz.

Funding Open Access funding enabled and organized by Projekt DEAL.

Open Access Dieser Artikel wird unter der Creative Commons Namensnennung 4.0 International Lizenz veröffentlicht, welche die Nutzung, Vervielfältigung, Bearbeitung, Verbreitung und Wiedergabe in 
jeglichem Medium und Format erlaubt, sofern Sie den/die ursprünglichen Autor(en) und die Quelle ordnungsgemäß nennen, einen Link zur Creative Commons Lizenz beifügen und angeben, ob Änderungen vorgenommen wurden.

Die in diesem Artikel enthaltenen Bilder und sonstiges Drittmaterial unterliegen ebenfalls der genannten Creative Commons Lizenz, sofern sich aus der Abbildungslegende nichts anderes ergibt. Sofern das betreffende Material nicht unter der genannten Creative Commons Lizenz steht und die betreffende Handlung nicht nach gesetzlichen Vorschriften erlaubt ist, ist für die oben aufgeführten Weiterverwendungen des Materials die Einwilligung des jeweiligen Rechteinhabers einzuholen.

Weitere Details zur Lizenz entnehmen Sie bitte der Lizenzinformation auf http://creativecommons.org/ licenses/by/4.0/deed.de.

\section{Literatur}

\section{Verwendete Literatur}

al-Awlaki, A. (2008, 11. Mai). Die Schlacht der Herzen und Köpfe, übersetzt von Umm Yayub. http://www.ahlu-sunnah.com/threads/22030-Die-Schlacht-der-Herzen-und-K\%C3\%B6pfe-Anwaral-Awlaki. Zugegriffen: 24. Aug. 2020.

Anzalone, C. (2010). Zachary Chesser: an American, grassroots jihadist strategist on raising the next generation of Al-Qaeda supporters. Perspectives on Terrorism, 4(5), 22-30.

Anzalone, C. (2012). The evolution of an American jihadi: the case of Omar Hammami. CTC Sentinel, 5(6), 10-13.

Baaken, T., \& Schlegel, L. (2017). Fishermen or swarm dynamics? Should we understand jihadist onlineradicalization as a top-down or bottom-up process? Journal for Deradicalization, 13, 178-211.

Baehr, D. (2019). Der Weg in den Jihad. Radikalisierungsursachen von Jihadisten in Deutschland. Wiesbaden: Springer VS.

Baugut, P., \& Neumann, K. (2019). Online propaganda use during Islamist radicalization. Information, Communication \& Society, 22, 1-23.

von Behr, I., Reding, A., Edwards, C., \& Gribbon, L. (2013). Radicalisation in the digital era. The use of the internet in 15 cases of terrorism and extremism. RAND Europe. https://www.rand.org/content/ dam/rand/pubs/research_reports/RR400/RR453/RAND_RR453.pdf. Zugegriffen: 4. Sept. 2020.

Bermingham, A., Conway, M., McInerney, L., O’Hare, N., \& Smeaton, A. F. (2009). Combining social network analysis and sentiment analysis to explore the potential for online radicalisation. International Conference on Advances in Social Network Analysis and Mining, 231-236. https://core.ac.uk/ download/pdf/147597985.pdf. Zugegriffen: 4. Sep. 2020.

Böckler, N., \& Allwinn, M. (2017). Hate - Open Source: Das Internet als Kontext für kollektive und individuelle Radikalisierungsprozesse. In N. Böckler \& J. Hoffmann (Hrsg.), Radikalisierung und extremistische Gewalt: Perspektiven aus dem Fall- und Bedrohungsmanagement (S. 233-257). Frankfurt a.M.: Verlag für Polizeiwissenschaft.

Böckler, N., Hoffmann, J., \& Meloy, R. J. (2017). “Jihad against the enemies of Allah”: the Berlin christmas market attack from a threat assessment perspective. Violence and Gender, 4(3), 73-82.

Böckler, N., Hoffmann, J., \& Zick, A. (2015). The Frankfurt airport attack: a case study on the radicalization of a lone-actor terrorist. Journal of Threat Assessment and Management, 2(3-4), 153-163.

Borum, R. (2011). Radicalization into violent extremism I: a review of social science theories. Journal of Strategic Security, 4(4), 7-36.

Bötticher, A., \& Mares, M. (2012). Extremismus. Theorien - Konzepte - Formen. München: Oldenbourg.

Bouhana, N., \& Wikström, P.-O. (2011). Al Qa'ida-influenced radicalisation: a rapid evidence assessment guided by situational action theory. Occasional paper 97. Home Office. https://assets.publishing. service.gov.uk/government/uploads/system/uploads/attachment_data/file/116724/occ97.pdf. Zugegriffen: 24. Aug. 2020.

Bowman-Grieve, L. (2013). A psychological perspective on virtual communities supporting terrorist and extremist ideologies as a toll for recruitment. Security Informatics, 2(9), 1-5.

Boyle, M. (2013). Lone wolf terrorism and the influence of the internet in France. CISLA Senior Integrative Project. https://digitalcommons.conncoll.edu/cgi/viewcontent.cgi article=1000\&context=sip. Zugegriffen: 24. Aug. 2020. 
Christoph, S. (2015). Funktionslogik terroristischer Propaganda im bewegten Bild. Journal for Deradicalization, 4, 145-205.

Conway, M. (2006). The terrorists use of the internet and fighting back. Information \& Security. An International Journal, 19, 9-30.

Conway, M. (2010). From al-Zarqawi to al-Awlaki: the emergence of the internet as a new form of violent radical milieu. CTX: Combating Terrorism Exchange, 2(9), 12-20.

Conway, M. (2016). Determining the role of the internet in violent extremism and terrorism: six suggestions for progressing research. Studies in Conflict and Terrorism, 39, 1-22.

Dienstbühl, D., \& Weber, M. (2014). Rekrutierung im Cyberspace - wie Extremisten das Internet nutzen. Journal for Deradicalization, 2, 35-45.

Ducol, B., Bouchard, M., Davies, G., Ouellet, M., \& Neudecker, C. (2016). Assessment of the state of knowledge: connections between research on the social psychology of the internet and violent extremism. TSAS working paper series 16-05. http:/www.tsas.ca/wp-content/uploads/2018/03/ TSASWP16-05_DucolEtAl.pdf. Zugegriffen: 24. Aug. 2020.

Frischlich, L., Rieger, D., Hein, M., \& Bente, G. (2015). Dying the right-way? Interest in and perceived persuasiveness of parochial extremist propaganda increases after mortality salience. Frontiers in Psychology: Evolutionary Psychology and Neuroscience, 6, 1-11.

Gill, P. (2015). Lone-actor terrorist. A behavioural analysis. Abingdon: Taylor \& Francis.

Gill, P., Horgan, J., \& Deckert, P. (2013). Bombing alone: tracing the motivations and antecedent behaviors of lone-actor terrorists. Journal of Forensic Sciences, 59(2), 425-435.

Gill, P., Corner, E., \& Conway, M. (2015). What are the roles of the internet in terrorism? Measuring online behaviors of convicted UK terrorists. VOX-Pol. https://www.voxpol.eu/download/vox-pol_ publication/What-are-the-Roles-of-the-Internet-in-Terrorism.pdf. Zugegriffen: 24. Aug. 2020.

Gill, P., Corner, E., Conway, M., Thornton, A., Bloom, M. \& Horgan, J. (2017). Terrorist Use of the Internet by the Numbers. Criminology \& Public Policy, 16(1), 99-117. https://doi.org/10.1111/17459133.12249.

Hack, K. (2009). The Malayan Emergency as Counter-Insurgency Paradigm. Journal of Strategic Studies, 32(3), 383-414.

Hamm, M. S., \& Spaaij, R. (2017). Paradigmatic case studies and prison ethnography: future directions in terrorism research. In G. LaFree \& J.D. Freilich (Hrsg.), Handbook of the Criminology of Terrorism (S. 206-220). Hoboken: John Wiley \& Sons Inc..

Inan, A. (2012). Jugendliche als Zielgruppe salafistischer Internetaktivität. In A. Toprak \& G. Weitzel (Hrsg.), Salafismus in Deutschland (S. 103-118). Wiesbaden: Springer VS.

Janbek, D., \& Prado, P. (2012). Rethinking the role of virtual communities in terrorist websites. CTX, 2(4), 23-27.

Jukschat, N., \& Kudlacek, D. (2017). Ein Bild sagt mehr als tausend Worte? Zum Potenzial rekonstruktiver Bildanalysen für die Erforschung von Radikalisierungsprozessen in Zeiten des Internets - eine exemplarische Analyse. In S. Hohnstein \& M. Herding (Hrsg.), Digitale Medien und politisch-weltanschaulicher Extremismus im Jugendalter. Erkenntnisse aus Wissenschaft und Praxis (S. 59-82). Deutsches Jugendinstitut. Arbeits- und Forschungsstelle Rechtsextremismus und Radikalisierungsprävention. https://www.dji.de/fileadmin/user_upload/bibs2017/Digitale_Medien.AFS.Band.13.pdf. Zugegriffen: 24. Aug. 2020

Kiefer, M., Hüttermann, J., Dziri, B., Ceylan, R., Roth, V., Srowig, F., \& Zick, A. (2018). „Lasset uns in sha'a Allah ein Plan machen": Fallgestützte Analyse der Radikalisierung einer WhatsApp-Gruppe (Islam in der Gesellschaft). Wiesbaden: Springer VS.

Knipping-Sorokin, R., \& Stumpf, T. (2018). Radikal Online - Das Internet und die Radikalisierung von Jugendlichen: eine Metaanalyse zum Forschungsfeld. kommunikation \& gesellschaft, 19, 1-29.

Landesamt für Verfassungsschutz Hamburg. (2012). Verfassungsschutzbericht 2011. https://www.hamburg. de/contentblob/3409076/4d4abeb4d97587160b7011c60f132317/data/verfassungsschutzbericht-2011nur-text-fassung-fhh.pdf;jsessionid=C4254AABDF615353B06FD9275A187954.liveWorker2. Zugegriffen: 4. Sep. 2020.

Leygraf, N. (2014). Zur Phänomenologie islamistisch-terroristischer Straftäter. Forensische Psychiatrie, Psychologie, Kriminologie, 8(4), 237-245.

Malthaner, S., \& Lindekilde, L. (2017). Analyzing pathways of lone-actor radicalization: a relational approach. In M. Stohl, S. Englund \& R. Burchill (Hrsg.), Constructions of terrorism (S. 163-180). Los Angeles: University of California Press.

Mastors, E., \& Siers, R. (2014). Omar al-Hammami: a case study in radicalization. Behavioral Sciences \& the Law, 32(3), 377-388. 
McCauley, C., \& Moskalenko, S. (2017). Understanding political radicalization: the two-pyramids model. American Psychologist, 72(3), 205-216.

Moghaddam, F. M. (2005). The staircase to terrorism: a psychological exploration. American Psychologist, $60(2), 161-169$.

Neumann, K. (2018). Medien und Islamismus. Der Einfluss von Medienberichterstattung und Propaganda auf islamistische Radikalisierungsprozesse. Wiesbaden: Springer VS.

Neumann, P.D., Winter, C., Meleagrou-Hitchens, A., Ranstorp, M., \& Vidino, L. (2018). Die Rolle des Internets und sozialer Medien für Radikalisierung und Deradikalisierung. PRIF Report 10/2018. Peace Research Institute, Leibniz-Institut Hessische Stiftung Friedens- und Konfliktforschung. https:// www.hsfk.de/fileadmin/HSFK/hsfk_publikationen/prif1018.pdf. Zugegriffen: 4. Sep. 2020.

O'Connor, F., Malthaner, S., \& Lindekilde, L. (2017). Killing in pairs: radicalisation patterns of violent dyads. International Journal of Conflict and Violence, 12, 1-12.

O’Connor, F., Malthaner, S., \& Lindekilde L. (2018). Killing in Pairs: Radicalisation Patterns of Violent Dyads. International Journal of Conflict and Violence, 12, 1-12.

Odağ, Ö., Leiser, A., \& Boehnke, K. (2020). Reviewing the role of the internet in radicalization processes. Journal for Deradicalization, 21, 261-300. 2019/2020.

Pearson, E. (2015). The case of Roshonara Choudhry: implications for theory on online radicalization, ISIS women, and the gendered jihad. Policy \& Internet, 7(1), 1-29.

Quiggin, T. (2010). Contemporary Jihadist narratives: The case of Momin Khawaja. In: National Coordinator for Counterterrorism. (Hrsg.), Countering violent extremist narratives (S. 84-93). https://www. clingendael.org/sites/default/files/pdfs/Countering-violent-extremist-narratives.pdf. Zugegriffen: 4. Sep. 2020.

Radlauer, D. (2007). Virtual communities as pathways to extremism. NATO Science for Peace and Security Series, 25, 67-75.

Ramelsberger, A. (2008). Der Deutsche Dschihad. Islamistische Terroristen planen Anschlag. Berlin: Eco. Rieger, D., Frischlich, L., \& Bente, G. (2013). Propaganda 2.0: psychological effects of right-wing and Islamic extremist internet videos. München: Luchterhand.

Rieger, D., Frischlich, L., \& Bente, G. (2019). Dealing with the dark side: the effects of right-wing extremist and Islamist extremist propaganda from a social identity perspective. Media, War \& Conflict, 13(3), 280-299.

Rogan, H. (2006). Jihadism Online - A study of how al-Qaida and radical Islamist groups use the Internet for terrorist purposes. Norwegian Defence Research Establishment (FFI). http://rapporter.ffi.no/ rapporter/2006/00915.pdf. Zugegriffen: 24. Aug. 2020.

Rogan, H. (2007). Al-Qaeda's online media strategies: from Abu Reuter to Irhabi 007. FFI. https:// publications.ffi.no/nb/item/asset/dspace:3401/07-02729.pdf. Zugegriffen: 24. Aug. 2020.

Roger, N. (2013). Image warfare in the war of terror. In S. Croft (Hrsg.), New security challenges. London: Macmillan.

Schmitt, J., Ernst, J., Frischlich, L., \& Rieger, D. (2017). Rechtsextreme und islamistische Propaganda im Internet: Methoden, Wirkungen und Präventionsmöglichkeiten. In R. Altenhof, S. Bunk \& M. Piepenschneider (Hrsg.), Politischer Extremismus im Vergleich (S. 171-210). Berlin: Lit.

Schwartz, D. (2012, 12. Juni). Supreme court reviews first anti-terrorism act case. CBC. https://www.cbc. $\mathrm{ca} /$ news/canada/supreme-court-reviews-first-anti-terrorism-act-case-1.1256918. Zugegriffen: 24. Aug. 2020.

Senate Committee on Homeland Security and Governmental Affairs. (2012). Zachary Chesser: a case study in online Islamist radicalization and its meaning for the threat of homegrown terrorism. https://www. hsdl.org/?view\&did=701274. Zugegriffen: 24. Aug. 2020.

Silber, M.D., \& Bhatt, A. (2007). Radicalization in the west: The homegrown threat. The New York City Police Department. https://seths.blog/wp-content/uploads/2007/09/NYPD_Report-Radicalization_ in_the_West.pdf. Zugegriffen: 24. Aug. 2020.

Ständige Konferenz der Innenminister und -senatoren der Länder. (2016). Analyse der Radikalisierungshintergründe und -verläufe der Personen, die aus islamistischer Motivation aus Deutschland in Richtung Syrien oder Irak ausgereist sind, aktualisierte Fassung vom Frühjahr 2016.

Steinberg, G. (2014). Al-Qaidas deutsche Kämpfer. Die Globalisierung des islamistischen Terrorismus. Hamburg: edition Körber-Stiftung.

Steinberg, G. (2012). Die Elif-Media-Informationsgruppe und die Deutschen Taliban Mujahidin. Stiftung Wissenschaft und Politik (SWP). https://www.swp-berlin.org/fileadmin/contents/products/studien/ 2012_S23_sbg.pdf. Zugegriffen: 24. Aug. 2020.

Stout, M.E. (2008). The terrorist perspectives project. Strategic and operational views of Al Qaida and associated movements. Annapolis: Naval Institute Press. 
SUNNAinDAhouse. (2009, 7. März). Das Mindeste was wir für unsere Ummah tun können. YouTube. Zugegriffen: 20. Apr. 2012.

Torres-Soriano, M. R. (2013). The dynamics of the creation, evolution, and disappearance of terrorist internet forums. International Journal of Conflict and Violence, 7(1), 164-178.

Vidino, L., \& Hughes, S. (2015). ISIS in America. From retweets to Raqqa. Program on extremism. The George Washington University. https://extremism.gwu.edu/sites/g/files/zaxdzs2191/f/downloads/ ISIS\%20in\%20America\%20-\%20Full\%20Report.pdf. Zugegriffen: 24. Aug. 2020.

Vidino, L., Kilberg, J., Lefkowitz, J., \& Kohlmann, E. (2015). Terrorist chatter. Understanding what terrorists talk about. Working paper No. 3. NPSIA University Carlton. https://core.ac.uk/download/pdf/ 217619308.pdf. Zugegriffen: 24. Aug. 2020.

Weimann, G. (2006a). Terror on the internet. The new arena, the new challenges. Washington: US Institute of Peace Press.

Weimann, G. (2006b). Virtual training camps: terrorist's use of the internet. In J. J.F. Forest (Hrsg.), Teaching terror, strategic and tactical learning in the terrorist world. Oxford: Rowman \& Littlefield.

Weimann, G. (2006c). Terrorist dot com: using the internet for terrorist recruitment and mobilization. In J. J. F. Forest (Hrsg.), Recruitment Bd. I. Westport: Praeger.

Weimann, G. (2006d). Virtual disputes: the use of the internet for terrorist debates. Studies in Conflict and Terrorism, 29, 623-639.

Whitlock, C. (2008, 24. Juni 2008). Al Qaeda's growing online offensive. Washington Post. http://www. washingtonpost.com/wpyn/content/article/2008/06/23/AR2008062302135.html. Zugegriffen: 10. Aug. 2015.

\section{Weiterführende Literatur}

Baehr, D. (2009). Kontinuität und Wandel in der Ideologie des Jihadi-Salafismus. Eine ideentheoretische Analyse der Schriften von Abu Mus'ab al-Suri, Abu Mohammad al-Maqdisi und Abu Bakr Naji. Bonn: Bouvier.

Baehr, D. (2011). Dezentraler, führerloser Jihad im Sinne al Suris? In A. Pfahl-Traughber (Hrsg.), Jahrbuch für Extremismus- und Terrorismusforschung (S. 516-548). Hochschule des Bundes für öffentliche Verwaltung. https://www.hsbund.de/SharedDocs/Downloads/2_Zentralbereich/20_ Referat_W/50_Publikationen/20_Schriften_Extremismus_Terrorismusforschung/band_06.pdf? blob=publicationFile \&v=4. Zugegriffen: 4. Sep. 2020.

Baehr, D. (2012). Salafistische Propaganda im Internet. Von der reinen Mission bis zum globalen Jihad. In S. Schuhmacher, \& J. W. Meine (Hrsg.), Magdeburger Journal zur Sicherheitsforschung, Bd. 2 (S. 236-269). http://www.sicherheitsforschung-magdeburg.de/journal_archiv.html. Zugegriffen: 24. Aug. 2020.

Baehr, D. (2014). Dschihadistischer Salafismus in Deutschland. In T. G. Schneiders (Hrsg.), Salafismus in Deutschland. Ursprünge und Gefahren einer islamisch-fundamentalistischen Bewegung. Bielefeld: transcript. 